\title{
Use of Normalization Process Theory to explore key stakeholders' perceptions of the facilitators and barriers to implementing electronic systems for medicines management in hospital settings
}

\author{
D. Hogan-Murphy ${ }^{\mathrm{a}, *}$, D. Stewart ${ }^{\mathrm{b}}$, A. Tonna ${ }^{\mathrm{a}}$, A. Strath ${ }^{\mathrm{a}}$, S. Cunningham ${ }^{\mathrm{a}}$ \\ ${ }^{\text {a }}$ Robert Gordon University, United Kingdom \\ ${ }^{\mathrm{b}}$ Qatar University, Qatar
}

\section{A R T I C L E I N F O}

\section{Keywords:}

Normalization process theory

eHealth

Medication

Implementation

Hospital

Healthcare professionals

\begin{abstract}
A B S T R A C T
Background: Limited data exist on the facilitators and barriers to implementing electronic systems for medicines management in hospitals. Whilst numerous studies advocate system use in improved patient safety and efficiency within the health service, their rate of adoption in practice has been slow.

Objective: To explore the perceptions of key stakeholders towards the facilitators and barriers to implementing electronic prescribing systems, robotic pharmacy systems, and automated medication storage and retrieval systems in public hospital settings using Normalization Process Theory as a theoretical framework.

Methods: Individual face-to-face semi-structured interviews were conducted in three public hospitals in Ireland with 23 consenting participants: nine nurses; four pharmacists; two pharmacy technicians; six doctors; and two Information Technology managers.

Results: Enhanced patient safety and efficiency in healthcare delivery emerged as key facilitators to system implementation, as well as the need to have clinical champions and a multi-disciplinary implementation team to promote engagement and cognitive participation. Key barriers included inadequate training and organisational support, and the need for ease and confidence in system use to achieve collective action.

Conclusions: Many themes that are potentially transferable to other national settings have been identified and extend the evidence base. This will assist organisations around the world to better plan for implementation of medication-related eHealth systems.
\end{abstract}

\section{Introduction}

Internationally there is widespread investment in eHealth, defined as "the exploitation of information and communication technologies (ICT) in healthcare to enhance the quality and safety of patient care". ${ }^{1}$ Several studies have identified that eHealth has the potential to ensure continuous improvements in healthcare and underpins organisational transformation and development. ${ }^{2}$ Medicines management in hospitals incorporates the entire process of how medicines are selected, procured, delivered, prescribed, administered, and reviewed to achieve informed and desired patient outcomes. Although the use of medicines is currently increasing in number and complexity ${ }^{3}$ which potentially amplifies medication error risks, systems for prescribing, dispensing, and administering medicines in Ireland and the UK have remained largely unchanged over the last few decades. ${ }^{4,5}$ Lack of system implementation may be due to organisational inefficiencies and the multi-level complexity of integrating new technology into existing work practices. The importance of adequate infrastructure and resources, managing expectations, and organisational readiness have been highlighted in systematic reviews on factors that promote and inhibit the implementation of eHealth systems. ${ }^{6,7}$ Limited attention was given to work directed at making sense of eHealth systems, effects on roles and responsibilities, methods of engaging with professionals, and ensuring potential benefits of implementation were apparent. ${ }^{6}$ In addition, a systematic review on healthcare professionals' perceptions of the facilitators and barriers to implementing electronic systems for prescribing, dispensing, and administering medicines in hospitals found team leadership and hardware/software availability and reliability were essential for successful implementation, and poor communication between healthcare professionals and patients a key barrier. ${ }^{8}$ The review also identified few studies have been published on this topic.

In Ireland, implementation of electronic systems for medicines in

\footnotetext{
* Corresponding author. Pharmacy Department, University Hospital Galway, Newcastle Road, Galway, H91YR71, Ireland.

E-mail addresses: diana.hogan-murphy@hse.ie, diana.hogan-murphy@hse.ie (D. Hogan-Murphy), d.stewart@qu.edu.qa (D. Stewart), a.tonna@rgu.ac.uk (A. Tonna), a.strath@rgu.ac.uk (A. Strath), s.cunningham@rgu.ac.uk (S. Cunningham).
} 
hospitals is further complicated by the immaturity of the information technology (IT) systems market, the variable levels of commercial and organisational expertise, and the overall limited investment in healthcare IT which accounts for one of the lowest levels in Europe. ${ }^{9,10}$ The Health Service Executive (HSE) provides and funds all public health services in hospitals and communities across Ireland. New structures are currently in the process of formation with the establishment of seven Hospital Groups as a transition to six new regional health areas and the government's overall commitment to reform the current highly criticised health service. ${ }^{10}$ Each with their own governance, management, and primary academic partner, the establishment of Hospital Groups is potentially a key enabler for reorganisation of services across hospitals with associated benefits of high quality patient care in a cost efficient manner. Support for eHealth adoption in recent years through publication of a national eHealth Strategy and development of national key building block initiatives to facilitate system implementation has been a positive progression and considerable interest has been expressed in the acquisition of these systems nationally. ${ }^{10,11}$ Initiatives include the establishment of eHealth Ireland, the ePharmacy agenda, and Health Information and Quality Authority (HIQA) standards around interoperability. HIQA is an independent authority that exists to improve health and social care services in Ireland. ${ }^{10}$

It is anticipated that system-users and implementers will be able to use this research when planning, implementing, maintaining, and sustaining these systems. Findings can then be used to improve the current system in hospitals and maximise the implementation and potential use of these systems in the future.

Gaps identified within the literature evolved into the overall novel aim of this research, namely to explore the perceptions of key stakeholders towards the facilitators and barriers to implementing electronic prescribing (ePrescribing), robotic pharmacy systems, and automated medication storage and retrieval systems in public hospital settings using Normalization Process Theory (NPT) as a theoretical framework to better understand processes involved in system adoption.

\section{Methods}

\section{Research design}

Conducting individual face-to-face semi-structured qualitative interviews with key stakeholders in three public hospital sites in Ireland underpinned by NPT was considered the most appropriate method to facilitate in-depth rich data capture and analysis. NPT focuses on work that individuals and organisations must perform for a new technology or practice to become embedded and sustained in routine practice and is used as a conceptual framework to explore the gap between health research evidence, policy, and practice. ${ }^{12}$ It concentrates on what people actually do rather than what they think and can be used to develop interview schedules, coding and analytical frameworks, and considers the interpretation and impact of research findings. The four constructs of NPT comprise: coherence (what is the work?), cognitive participation (who does the work?), collective action (how does the work get done?), and reflexive participation (how is the work understood?). ${ }^{13}$ These constructs are operationalised under 16 components, as described in Table 1. A qualitative systematic review by McEvoy et al., in 2014 using NPT to research implementation processes identified 29 papers and found coherence and cognitive participation relate more to the 'planning' stages of implementation, and collective action and reflexive monitoring relate more to 'experiences' post implementation. ${ }^{11}$ Two interview schedules were therefore developed underpinned by NPT and existing literature by all members of the research team for participants working in hospitals with and without system implementation. Subsequently, a more recent qualitative systematic review by May et al., in 2018 using NTP in feasibility studies and process evaluations of complex healthcare interventions identified 130 papers. ${ }^{14}$ They found NPT was useful across a wide range of interventions and was more frequently operationalised and embedded in study protocols.

\section{Setting}

Public hospitals provided by the HSE were selected as they cover the majority of hospital types in Ireland and are guided by national eHealth programmes and availability of government funding which impacts on decisions to invest in eHealth systems. At the time of this study, no public sector hospital in Ireland had implemented a hospital-wide ePrescribing system linking prescriptions electronically between prescribers and dispensers, one public hospital had introduced a robotic pharmacy system, and three public hospitals had implemented automated medication storage and retrieval systems.

\section{Participant sample}

To capture a broad range of perspectives from participants with and without system experience and facilitate data analysis across different hospital sites and diversity in terms of maturity of system implementation, two general hospitals in the public sector that had introduced automated medication storage and retrieval systems at different implementation stages (over 10 years and seven months), and one general hospital in the public sector which was considering implementation were selected. Purposive sampling was employed in order to identify a range of relevant key stakeholders for participation. The main stratification factors employed were potential key implementers and operational end-users perceptions before system adoption with no previous experience; key implementers and operational end-users' perceptions and experiences after system implementation; profession; and grade. The research team felt including participants without experience was equally as important as including participants with experience in order to identify perceived facilitators and barriers prior to implementation and understand its likely impact, success, and sustainability.

\section{Sample size}

An initial sample size of 24 key stakeholders were invited to participate and share their perceptions of the facilitators and barriers to implementing ePrescribing, robotic pharmacy systems, and/or automated medication storage and retrieval systems. As per previous research conducted on adequate sample size, this number was expected to capture a broad variety of perceptions towards system implementation and assist in identifying common and diverse themes and reach both meaning and code saturation. ${ }^{15,16}$

\section{Development of interview schedules}

Mapping of NPT constructs to the interview schedules is provided in Table 2. Particular attention was paid to Creswell's key recommendations in developing the interview schedules, as provided in Table $3^{17}$. The schedules were further reviewed for credibility by five experts with vast experience in the topic under investigation, and piloted with a pharmacy technician, nurse, and doctor. The interview schedules were modified with minor changes thereafter as per feedback offered.

\section{Data generation}

Interviews were conducted post informed consent between February and March 2016 in the hospital setting of inquiry, audio-recorded, and transcribed verbatim by the primary researcher (DHM). Participants were provided an opportunity to review their own interview transcript for further credibility prior to two members of the research team (SC and AT) reviewing three transcripts each for robustness. No significant discrepancies were identified. 
Table 1

NPT constructs and components applied to this research adopted from Mair et al. ${ }^{6}$

\begin{tabular}{|c|c|c|c|}
\hline Coherence (Sense-making work) & $\begin{array}{l}\text { Cognitive participation } \\
\text { (Relationship work) }\end{array}$ & Collective action (Enacting work) & Reflexive monitoring (Appraisal work) \\
\hline Differentiation & Enrolment & Skill set workability & Reconfiguration \\
\hline $\begin{array}{l}\text { Is there a clear understanding of how the new } \\
\text { eHealth system differs from existing practice? }\end{array}$ & $\begin{array}{l}\text { Do individuals buy-in to the idea } \\
\text { of the eHealth system? }\end{array}$ & $\begin{array}{l}\text { How does the eHealth system affect } \\
\text { roles and responsibilities or training } \\
\text { needs? }\end{array}$ & Do individuals try to alter the new service? \\
\hline Communal specification & Activation & Contextual Integration & Communal appraisal \\
\hline $\begin{array}{l}\text { Do individuals have a shared understanding of the } \\
\text { aims, objectives, and expected benefits of the } \\
\text { eHealth system? }\end{array}$ & $\begin{array}{l}\text { Can individuals sustain } \\
\text { involvement? }\end{array}$ & Is there organisational support? & $\begin{array}{l}\text { How do groups judge the value of the eHealth } \\
\text { system? }\end{array}$ \\
\hline Individual specification & Initiation & Interactional workability & Individual appraisal \\
\hline $\begin{array}{l}\text { Do individuals have a clear understanding of their } \\
\text { specific tasks and responsibilities in system } \\
\text { implementation? }\end{array}$ & $\begin{array}{l}\text { Are key individuals willing to } \\
\text { drive implementation? }\end{array}$ & $\begin{array}{l}\text { Does the eHealth system make } \\
\text { people's work easier? }\end{array}$ & $\begin{array}{l}\text { How do individuals appraise the effects of the } \\
\text { eHealth system on them and their work } \\
\text { environment? }\end{array}$ \\
\hline Internalisation & Legitimation & Relational integration & Systematization \\
\hline $\begin{array}{l}\text { Do individuals understand the value, benefits, and } \\
\text { importance of the eHealth system? }\end{array}$ & $\begin{array}{l}\text { Do individuals believe it is right } \\
\text { for them to be involved? }\end{array}$ & $\begin{array}{l}\text { Do individuals have confidence in the } \\
\text { new system? }\end{array}$ & $\begin{array}{l}\text { How are benefits or problems identified or } \\
\text { measured? }\end{array}$ \\
\hline
\end{tabular}

\section{Data management and analysis}

Data were analysed using the framework approach to generate a set of a priori codes. This systematic approach has been applied to other healthcare studies with $\mathrm{NPT}^{18-21}$ and is used to categorise data into emerging themes in a hierarchical order through five interrelated stages: familiarisation; identifying a thematic framework; indexing; charting; and mapping and interpretation. ${ }^{22}$ Familiarisation involved the primary researcher transcribing the interviews verbatim and reading the transcriptions repeatedly whilst listening to the digital audio recordings. A thematic framework was identified by developing themes from re-reading the interview transcripts and highlighting significant quotes. Key words from the research objective identified some thematic codes as well as the recurrent themes from the transcripts. QSR NVivo11 ${ }^{\circledR}$ qualitative data management software facilitated the sorting of codes during the indexing stage of data analysis. Charting was created by connecting the thematic codes according to how they related to each other by either merging or reducing themes. Mapping and interpretation involved searching for patterns, associations, concepts, and explanations of the data using verbatim quotes to illustrate themes. All transcripts were reviewed for emerging themes by DHM and then divided equally between SC and AT for independent coding and analysis. No significant discrepancies were identified. All data were anonymised, coded, and securely stored. Ethical approval was received from a UK university where the research was carried out and all participating hospitals' ethics committees prior to conducting the interviews.

\section{Results}

Twenty-three of the 24 invitees participated: nine nurses, six pharmacy staff, six doctors, and two IT managers, equally divided per hospital site. An IT professional declined to be interviewed without providing a reason. The median time for interviews was 38 minutes.
Table 3

Key recommendations for developing interview questions in qualitative research adopted from Creswell et al. ${ }^{17}$

- Ask no more than five to seven sub-questions in addition to central questions

- Relate the central questions to the specific qualitative strategy of inquiry

- Begin the research questions with the words 'what' or 'how' to convey an open and emerging design

- Focus on a single phenomenon or concept

- Use exploratory verbs that convey the language of emerging design, use nondirectional rather than directional words

- Expect the research questions to evolve and change during the study in a manner consistent with the assumptions of an emerging design

- Use open-ended questions without reference to the literature or theory unless otherwise indicated by a qualitative strategy of inquiry

- Specify the participants and research site for the study if not already provided

Meaning and code saturation were deemed to have occurred by DHM, SC, and AT and no new themes emerged in terms of thematic ranges. Further recruitment was not undertaken. The median years of professional work experience was 16-20 years and the majority of participants had practised outside of Ireland and had system experience, as provided in Table 4. Doctors mostly discussed ePrescribing systems, nurses and IT professionals mostly discussed automated medication storage and retrieval systems, and pharmacy participants mostly discussed automated medication storage and retrieval systems and robotic pharmacy systems. Eight key themes emerged from data analysis using NTP as a theoretical framework, as summarised in Table 5.

Theme 1: Understanding of how electronic systems differ from manual practices and the value of system implementation

Participants had a clear understanding of the aim of implementation, with key concepts of enhanced patient safety and efficiency

Table 2

Mapping of concepts in the interview schedules to NPT.

Interview schedule concepts

\section{Coherence}

Sense-making work that people do individually and collectively at the planning stages of implementation

Cognitive participation

Relational work that people do to enrol and engage with the planning of implementation

Collective action

Operational work that people do to enact the new system

Perceptions of the overall goals of implementation e.g. patient safety, increased efficiency

Responsibility for implementation e.g. implementers driving it forward, end-users buyin to implementation

Tasks carried out in delivering the implementation process e.g. training, policies 
Table 4

Characteristics of study participants.

\begin{tabular}{|c|c|}
\hline Characteristics & $\mathrm{n}$ \\
\hline \multicolumn{2}{|l|}{ Sex } \\
\hline Female & 16 \\
\hline Male & 7 \\
\hline \multicolumn{2}{|l|}{ Healthcare professionals } \\
\hline Doctors & 6 \\
\hline Nurses & 9 \\
\hline Pharmacists & 4 \\
\hline Pharmacy technicians & 2 \\
\hline Hospital IT managers & 2 \\
\hline \multicolumn{2}{|l|}{ Grade } \\
\hline Senior & 15 \\
\hline Junior & 8 \\
\hline \multicolumn{2}{|l|}{ Years of experience in profession } \\
\hline$<1$ year & 1 \\
\hline $1-5$ years & 2 \\
\hline $6-10$ years & 2 \\
\hline $11-15$ years & 3 \\
\hline $16-20$ years & 5 \\
\hline $21-25$ years & 4 \\
\hline $26-30$ years & 2 \\
\hline $31-35$ years & 1 \\
\hline$>35$ years & 3 \\
\hline Practised in countries outside Ireland & 13 \\
\hline \multicolumn{2}{|l|}{ Countries: } \\
\hline United Kingdom & 9 \\
\hline Jersey Island & 1 \\
\hline Pakistan & 1 \\
\hline New Zealand & 1 \\
\hline Australia & 1 \\
\hline Experience of using/implementing ePrescribing & 4 \\
\hline Experience of using/implementing robotic pharmacy systems & 1 \\
\hline $\begin{array}{l}\text { Experience of using/implementing automated medication storage and } \\
\text { retrieval systems }\end{array}$ & 13 \\
\hline
\end{tabular}

evident. Legibility of prescriptions, clinical decision support (CDS), accurate drug selection, and reduced medication errors were perceived to improve patient safety with implementation.

"There would be less errors in terms of not being able to read what the prescription is and the doses...I think safety has to be the biggest value you can get from it". (Senior nurse N5, no prior experience)

Stock control, traceability, accountability, cost containment, and integration of systems were other perceived benefits.

"If you have the systems right the way through from prescribing to dispensing, then you should have a continuous log that is retrievable". (Consultant doctor D6, prior experience)

Participants expressed concern over possible negative variations between electronic and manual systems such as potential time inefficiencies, security issues, and logistics of changing from manual to automatic.

"Any electronic system, it doesn't matter how streamlined you put it, it will be a hindrance because it will slow down processes". (Consultant doctor D5, no prior experience)

Theme 2: A need to work together to build a shared sense of purpose for system implementation and have a clear understanding of individual roles and responsibilities

Participants perceived that different professionals had differing ideas of the purpose of the system and that some individuals would work together to build a shared understanding of the reasons for implementation, and others would not. A perception of limited communication with colleagues on implementation resulted in participants either unable to determine if there was a shared sense of purpose or believing there was not enough information available to have a comprehensive shared understanding.

"I don't know because I haven't spoken to my working colleagues about it". (Nurse manager N2, no prior experience)

Participants with system experience had a clear understanding of their roles, including responsibility for planning and monitoring implementation, delivering adequate training to end-users, and becoming familiar with policies and protocols. Participants without system experience had limited understanding of what was required for implementation.

"It is all theory to me, I know vaguely what electronic prescribing is but how actually it works, I don't, it might be a more arduous task, I don't know yet. So that is the fear I suppose". (Senior nurse N5, no prior experience)

Theme 3: A need for clinical leadership, champions at ward level, and a multi-disciplinary implementation team to promote 'buy in'

A multi-disciplinary team approach, clinical leadership and champions at ward level were key concepts perceived to promote engagement with system implementation. Selecting early adaptors was also believed to be of benefit.

"I think maybe having champions at ward level, where they are involved in all pre discussions and planning meetings...try and get protected time for nursing to be part of the project implementation group to be more involved in the policies and reviewing what would work well for their ward." (Senior pharmacist P1, prior experience)

Participants felt the younger generation could realise the benefits more easily and that good communication and information sessions on the benefits of system implementation were mechanisms of promoting engagement.

"Having a working group that actively promote it and look at the advantages and just keep reminding people, it is brain washing really". (Senior nurse N5, no prior experience)

Resistance to work practice changes, force of change in practice, and limited involvement with end-users were viewed as barriers to active participation. Bureaucracy and lack of prioritisation for implementation were other barriers verbalised.

"There is a huge culture of resistance, people here are used to doing their own thing". (IT manager IT1, no prior experience)

Theme 4: A need for adequate training and organisational support

Small group hands-on training sessions, super-user support, training in areas only applicable to the user, phased training per ward, and sufficient time to train and adjust to new work practices were viewed as beneficial. Resource investment and robust governance inclusive of developing and disseminating policies and protocols, contingency plans, and completing risk assessments and competency assessments were also perceived as facilitators to successful system implementation. Participants' perceived operational guidelines assisted with supporting system implementation and understanding the effects of the new system on individuals' roles and responsibilities and training needs.

"Omnicell gave us their operational guidelines and then we drafted our own local guidelines where we outline the roles and responsibilities for all staff, from medical staff, nursing staff, pharmacy staff, IT staff, and then the company trainer and the out of hours support". (Senior pharmacist $\mathrm{P} 1$, prior experience)

A number of participants felt the training received was not sufficient, in particular where staff were not trained during initial implementation. 
Table 5

Summary of key facilitator and barrier themes related to NPT constructs and components.

\begin{tabular}{|c|c|c|c|}
\hline Key themes & $\begin{array}{l}\text { NPT constructs and } \\
\text { components }\end{array}$ & Facilitators & Barriers \\
\hline $\begin{array}{l}\text { Theme 1: } \\
\text { Understanding of how electronic systems differ from } \\
\text { manual practices and the value of system } \\
\text { implementation }\end{array}$ & $\begin{array}{l}\text { Coherence: } \\
\text { Differentiation } \\
\text { Internalisation }\end{array}$ & $\begin{array}{l}\text { Patient safety } \\
\text { Efficiency: } \\
\text { - Stock control } \\
\text { - Traceability } \\
\text { - Accountability } \\
\text { - Cost reduction } \\
\text { - Integration }\end{array}$ & $\begin{array}{l}\text { Time inefficiencies } \\
\text { Security issues } \\
\text { Logistics of changing system }\end{array}$ \\
\hline $\begin{array}{l}\text { Theme 2: } \\
\text { A need to work together to build a shared sense of } \\
\text { purpose for system implementation and have a clear } \\
\text { understanding of individual roles and responsibilities }\end{array}$ & $\begin{array}{l}\text { Coherence: } \\
\text { Communal specification } \\
\text { Individual specification }\end{array}$ & $\begin{array}{l}\text { Work together to understand reasons } \\
\text { for implementation } \\
\text { Participants with experience had a } \\
\text { clear understanding of their roles }\end{array}$ & $\begin{array}{l}\text { Limited communication on implementation } \\
\text { Participants without experience had a limited } \\
\text { understanding of requirements }\end{array}$ \\
\hline $\begin{array}{l}\text { Theme 3: } \\
\text { A need for clinical leadership, champions at ward } \\
\text { level, and a multidisciplinary implementation team to } \\
\text { promote buy-in }\end{array}$ & $\begin{array}{l}\text { Cognitive } \\
\text { participation: } \\
\text { Enrolment } \\
\text { Activation } \\
\text { Initiation } \\
\text { Legitimation }\end{array}$ & $\begin{array}{l}\text { Clinical champions to promote } \\
\text { benefits and engagement via effective } \\
\text { communication } \\
\text { Early adaptors } \\
\text { Multidisciplinary team approach }\end{array}$ & $\begin{array}{l}\text { Older generation may not realise benefits as } \\
\text { easily as younger generation } \\
\text { Resist work changes: } \\
\text { - Lack of prioritisation } \\
\text { - Force of change } \\
\text { - Limited involvement } \\
\text { - Bureaucracy } \\
\text { - Lack of recognition of professional roles }\end{array}$ \\
\hline $\begin{array}{l}\text { Theme 4: A need for adequate training and organisational } \\
\text { support }\end{array}$ & $\begin{array}{l}\text { Collective action: } \\
\text { Skill set workability } \\
\text { Contextual integration }\end{array}$ & $\begin{array}{l}\text { Sufficient training } \\
\text { Sufficient support and resources } \\
\text { Robust governance } \\
\text { Operational guidelines }\end{array}$ & $\begin{array}{l}\text { Training not sufficient } \\
\text { Inadequate support } \\
\text { No additional resources }\end{array}$ \\
\hline $\begin{array}{l}\text { Theme 5: A need for electronic systems to be easier to use } \\
\text { than manual systems }\end{array}$ & $\begin{array}{l}\text { Collective action: } \\
\text { Interactional } \\
\text { workability }\end{array}$ & $\begin{array}{l}\text { Light guided } \\
\text { Ease of stock management } \\
\text { Sufficient number of systems } \\
\text { Mobile units nearer the patient }\end{array}$ & $\begin{array}{l}\text { Manual system easier as more patient-focused and } \\
\text { less task oriented } \\
\text { Workflow issues e.g. time delays in queuing, } \\
\text { limited accessibility, inadequate numbers/sizes of } \\
\text { units resulting in delayed medication } \\
\text { administration }\end{array}$ \\
\hline Theme 6: A need for a sense of confidence in system use & $\begin{array}{l}\text { Collective action: } \\
\text { Relational integration }\end{array}$ & $\begin{array}{l}\text { Safety alerts } \\
\text { Double checking } \\
\text { Clear record } \\
\text { Confident with familiarity }\end{array}$ & $\begin{array}{l}\text { Lack of confidence with identifying drugs } \\
\text { Substantial time away from patients }\end{array}$ \\
\hline Theme 7: A need to use systems as intended & $\begin{array}{l}\text { Reflexive monitoring: } \\
\text { Reconfiguration }\end{array}$ & $\begin{array}{l}\text { Alter system use for efficiency e.g. } \\
\text { recheck chart before administration }\end{array}$ & $\begin{array}{l}\text { Not using system as trained e.g. trolley to carry } \\
\text { drugs for multiple patients increasing risk of } \\
\text { errors }\end{array}$ \\
\hline Theme 8: A need to measure and audit practice & $\begin{array}{l}\text { Reflexive monitoring: } \\
\text { Communal appraisal } \\
\text { Individual appraisal } \\
\text { Systematization }\end{array}$ & $\begin{array}{l}\text { Auditing of practice e.g. cost, time, } \\
\text { end-user satisfaction }\end{array}$ & $\begin{array}{l}\text { Limited formal measures } \\
\text { Unable to determine actuality from reality }\end{array}$ \\
\hline
\end{tabular}

"Very much the new staff are told 'this is how you log in' and then it is very much the staff on the ward will say 'this is what you need to do". (Nurse manager N4, prior experience)

Participants perceived inadequate management support and resources were provided and little consideration to the effects of system implementation on work practices. It was felt more engagement would have resulted in more responsibility and acceptance of system use.

"I think initially it was very much this is just something you are going to do and it was never really given the amount of thought of how much this was going to change the way the ward worked. So in terms of nursing support I don't feel there was a great deal there". (Junior nurse N8, prior experience)

Theme 5: A need for electronic systems to be easier to use than manual systems

Enhanced efficiency in relation to stock availability was perceived as a facilitator by participants.

"It is a huge turn around and they see the advantages and the time that was wasted every day for nurses sending down requisitions and the pharmacist ringing back questioning it and there was a whole conversation going on". (Nurse manager N9, prior experience)

However, nurses felt the manual system was easier to use and more patient focused and interactive. The new system was viewed as more task oriented than the traditional manual system.

"It is going back to a task, we got to go and get the drugs from the machine, so it is a task, but before there was more of a subtle dynamic in it and maybe we weren't even as aware of it. The drugs were very linked with patients, you had the visual cues". (Nurse manager N6, prior experience)

Work flow issues and time delays in queuing to remove drugs resulting in patients waiting for medication were viewed as substantial barriers to system compatibility with existing practices. This was mainly due to inadequate numbers and sizes of units impacting on administering medication as prescribed, retrieving medications in an emergency, and discharging patients. Further delays in inputting controlled drugs and pharmacy stocking the machine limiting accessibility were also viewed as frustrating. Instalment of additional systems and mobile units nearer the patient was a perceived requirement.

"You might have 31 patients to get their medicines for around that time with one point of access. Previously on the ward we would have had at minimum six points of access. We need more systems in place". (Nurse manager N6, prior experience)

Pharmacy participants also believed the new system was more time consuming and involved more work than the manual system.

"It is more work with Omnicell without a doubt. Even the time it takes to put stuff away. What happens if somebody puts the wrong thing in the 
wrong place, which can happen easily? So for a long time two people were going up and putting away the top up." (Senior pharmacist P5, prior experience)

\section{Theme 6: A need for a sense of confidence in system use}

Safety alerts such as therapeutic drug monitoring and administration instructions, two people double checking stocked items in the machine, and comprehensive records of retrieved medication enhanced a sense of confidence and accountability in using the system. In particular, a sense of confidence was evident when individuals became more familiar with the system.

"I was having trouble reading a drug kardex as I often do and I went back to look what the person previously gave and they had given what to me it said". (Junior nurse N8, prior experience)

Overall, expectations were not met with system implementation.

"We believed we were going to have this great pharmacy system and that every medication we wanted was going to be in it and there would be no delays administering drugs, there would be no delay in getting medication and it would be a safe system...so now we spend a lot more time away from the patient, getting medication for them and then the problem is once you leave that area, you're pulled at for loads of other things. It is very distracting cause it takes your focus away for possibly 10 or 15 minutes...it hasn't helped nursing in relation to one to one care with patients. (Nurse manager N4, prior experience)

\section{Theme 7: A need to use systems as intended}

Nursing and pharmacy participants felt they reflected on work practices and altered system use accordingly for efficiency purposes. This included discontinuing preordering of drugs, more night time ordering of drugs, rechecking the medication chart at the bedside before drug administration, and altering the method items were double checked by pharmacy technicians when stocking the system.

"Now we make sure that we check the drug kardexes again at the bedside, we had discussed that before, just to try and reduce errors". (Junior nurse N1, prior experience)

Various participants felt some individuals did not use the system as trained, such as removing more medication than requested leading to inaccuracies in drug amounts in the machine in comparison to what was reported.

"It is not fool proof, you can find a way round it, so if you go in for Panadol, you can take out two or three and tell it you took out one...we do know where stock is in theory, but we are still relying on people to remove things as they are supposed to." (Senior pharmacist P5, prior experience)

Other alterations included accessing pharmacy outside of opening hours for drugs already stocked in the machine, gaining access to prohibited functions of the system, and storing stocked medication outside the machine. The use of a trolley to carry drugs for multiple patients at one time was perceived as increasing the risk of errors and accessing the system for long periods.

"In A\&E they go from system to patient, but on the medical wards they use trolleys, so they remove the meds, put it into a specific trolley with specific drawers for the patients, so they could hold up the system for maybe an hour... there is an increased risk of errors, it should be system to patient but this is not the case because of the size of the medical wards and only having one machine". (Senior pharmacy technician P6, prior experience)

Lack of using the system to its maximum benefit was also perceived as a disadvantage, such as availing of CDS and integration of systems.

"I think there are a lot more capabilities that we have yet to implement and there is also the possibility of linking other systems into it". (Senior pharmacist $\mathrm{P} 1$, prior experience)

\section{Theme 8: A need to measure and audit practice}

Whilst not many formal methods of measuring the impact of system implementation were in place, reviewing financial reports, complaints, stock counts, and medication waste were believed to be effective ways of identifying facilitators and barriers with implementation.

"We did a financial report in pharmacy in a three month period prior to the systems being installed and a three month period after and there was a cost saving of between 15 and 17\%". (Senior pharmacy technician P6, prior experience).

Auditing of practice was perceived as another way of identifying benefits or problems and in understanding the systems value and requirements for future improvements. This included time comparisons between the new and old system, end-user perceptions before and after system implementation, error rates, and level of training.

"There was an audit done in the last few weeks and currently we are spending more time on the Omnicell than we would with the manual top up system, but that is because we have two members of staff going to the ward to fill the Omnicell so I think that is an issue around training as well so the managers plan is to get that down to one person". (Junior pharmacy technician P2, prior experience)

Measuring and auditing practice were also viewed as important in determining actuality rather than perceptions.

"You have always got to bear in mind, what staff sometimes say isn't always the reality, it could be a perception rather than the reality so that is why we have to bring in more measurement to see is it actually what is happening or is it what they think is happening so I have to do that, the two could be different". (Nurse manager N6, prior experience)

\section{Discussion}

This is the first published qualitative study to explore the facilitators and barriers specific to implementing electronic systems for prescribing, dispensing, and administering medicines in hospitals. Findings from implementers and end-users' perceptions of system adoption draw attention to issues around implementation which are multi-factorial and complex at both an individual level and organisational level. Findings derived through analysis of a systematic review on eHealth implementation were similar to findings from this research. ${ }^{8}$ Healthcare professionals perceived systems improved patient safety and provided better access to patients' drug records and that team leadership and hardware/software availability and reliability were essential for successful implementation. Key barriers included hardware and network problems, altered work practices, and weakened interpersonal communication between healthcare professionals and with patients.

Demonstration of coherence and cognitive participation were key drivers for success or failure at the initial stages of implementation. It was clear that individuals would engage and 'buy in' to implementation if the system was viewed as beneficial in improving work practices. Although some participants perceived key individuals' were willing to drive implementation, force of change in practice, limited involvement, and lack of understanding of the impact of implementation on services were evident. Negative attitudes acted as obstacles to enrolment, such as beliefs that the system would disrupt the delivery of care, distrust in system use, and a culture of resistance to change. This is similar to findings from Travaglia et al. who identified an initial failure to display coherence and cognitive participation resulting in end-users not perceiving the new way of working as helpful and relevant and an 
unwillingness to engage with the process. ${ }^{23}$

A range of strategies to initiate and legitimise system participation in this study included fostering a culture of clinical champions and selecting early adaptors for implementation with the support of a multidisciplinary team. Hardeep and colleagues also found a well designed project, multi-disciplinary approach, and ongoing engagement facilitated a smooth transition from paper to electronic systems in a UK hospital. ${ }^{24} \mathrm{~A}$ similar study found nurses should have a significant role in system design to ensure a smooth transition to system use. ${ }^{25}$

Further operational work and investment in resources and ongoing staff, contingency, and policy support were needed by individuals and organisations to enhance implementation processes and facilitate collective action, particularly with the nursing profession. Providing a period of transition in which end-users can become familiar with and learn how to use the new system was also required. In terms of ease of use and confidence in the new system, resistance was evident due to perceived added complexity, effort, and time. In particular, workflow issues with time away from patients, additional interruptions, and accessibility issues ultimately impinged on delayed administration of medication to patients. Offering a more complex and realistic picture at the initial stages of system implementation may manage future user expectations. In line with other research, findings from this study highlight the challenges of integrating new systems with existing work processes and the introduction of new risks, ${ }^{6-8,26}$ even when organisations have moved into more routine system use. ${ }^{27}$

Redwood and colleagues found the introduction of an ePrescribing system in a UK hospital had the potential to give rise to new types of risks to patient safety. ${ }^{26}$ These included pick list juxtaposition errors, confusion with paper based and electronic systems, and distractions and interruptions to workflows. Participants in this study felt medicines management would improve with the instalment of additional units nearer the patient. Incorporating workflow analysis into system design, integration of systems into the usual process of care, and minimising workflow interruptions were required to facilitate successful implementation. Participants understood ways of appraising the new system post implementation to identify facilitators and barriers and consider its effects on work practices. Concerns with system usability led to the development of workarounds by end-users. Cresswell and colleagues also found informal practices were employed by end-users not approved by management due to perceived changes to professional roles, issues with system usability and performance, and challenges relating to inaccessibility of hardware. ${ }^{28}$ Unintended errors and applying technology in ways other than intended have been further documented in a UK report on challenges and lessons learnt from ePrescribing in UK hospitals. ${ }^{4}$ As limited formal methods of reviewing benefits and issues post implementation were identified in this study, it is important to note that key themes which emerged within NPT are only participants' perceptions and may not align with actuality. It is also possible that some benefits such as time savings may have been masked by other frustrations arising from complex work processes. A need to promote reflexive monitoring to evaluate the outcomes of system implementation on patient care and workflows was evident.

The use of NPT has highlighted individual and organisational facilitators and barriers to the normaliation of these complex electronic systems into routine work which requires consideration to interventions inclusive of engagement, education, training, and support. Findings generated strongly emphasise the need for coherence, cognitive participation, collective action, and reflexive monitoring. Key facilitators included enhanced patient safety and efficiency and key barriers of workflow issues. Assessing and fostering readiness for technological innovation also appears to be particularly important for successful adoption. Many potentially transferable themes have been identified and extend the evidence base. This will assist organisations to better plan for implementation of medication-related eHealth systems.

\section{Conclusions}

Novel knowledge and understanding with regard to perceptions of key stakeholders towards the facilitators and barriers of implementing ePrescribing, robotic pharmacy systems, and automated medication storage and retrieval systems in hospitals in Ireland has been generated. The mix of participants comprising senior and junior nurses, pharmacy staff, doctors, and hospital IT managers with and without system experience perceived enhanced patient safety and efficiency as key facilitators to system implementation. They also felt the need to have clinical champions and a multidisciplinary implementation team to promote engagement and cognitive participation. Key barriers included inadequate training and organisational support, and the need for ease and confidence in system use to achieve collective action. Integrating new ways of working was perceived as challenging, mainly due to difficulties in understanding the complexity of implementing electronic systems at both an individual level, such as education, training, and defined roles, and an organisational level, such as allocation of resources and ongoing support. A systematic approach and further consideration to system implementation is required. Reviews have highlighted papers are generally of poor quality and issues of adoption multifactorial. There may then be value in employing standardised processes such as NPT in eHealth implementation. Further work could also focus on ethnographic methods to better understand current use and system workarounds.

\section{References}

1. Health Information and Quality Authority. Developing National eHealth Interoperability Standards for Ireland: A Consultation Document. Dublin: Health Information and Quality Authority; December 92011.

2. Murray E, Burns J, May C, et al. Why is it difficult to implement e-health initiatives? A qualitative study. Implement Sci. 2011;6:6-17.

3. Jyrkkä J, Vartiainen L, Hartikainen S, Sulkava R, Enlund H. Increasing use of medicines in elderly persons: a five-year follow-up of the Kuopio $75+$ Study. Eur J Clin Pharmacol. 2006;62(2):151-158.

4. National Health Service Connecting for Health. Electronic Prescribing in Hospitals Challenges and Lessons Learned. Leeds: National Health Service Connecting for Health; June 12009.

5. The Pharmaceutical Society of Ireland. The Pharmaceutical Society of Ireland Baseline Study of Hospital Pharmacy in Ireland. Dublin: The Pharmaceutical Society of Ireland; December 2012.

6. Mair FS, May C, O'Donnell C, Finch T, Sullivan F, Murray E. Factors that promote or inhibit the implementation of e-health systems: an explanatory systematic review. Bull World Health Organ. 2012;90(5):357-364.

7. Ross J, Stevenson F, Lau R, Murray E. Factors that influence the implementation of ehealth: a systematic review of systematic reviews (an update). Implement Sci. 2016;11(1):146-158.

8. Hogan-Murphy D, Tonna A, Strath A, Stewart D, Cunningham S. Healthcare professionals' perceptions of the facilitators and barriers to implementing electronic systems for prescribing, dispensing and administration of medicines in hospitals: a systematic review. Eur J Hosp Pharm. 2015;22:358-365.

9. Joint Research Centre - Institute for Prospective Technological Studies. European Hospital Survey: Benchmarking Deployment of Ehealth Services (2012-2013). Luxembourg: Publications Office of the European Union; March 102014

10. Department of Health. eHealth Strategy for Ireland. Dublin: Department of Health December; 132013.

11. McEvoy R, Ballini L, Maltoni S, O'Donnell CA, Mair FS, MacFarlane A. A qualitative systematic review of studies using the normalization process theory to research implementation processes. Implement Sci. 2014;9:2-12.

12. Normalization process theory on-line user's manual and toolkit. Online referencing: http://www.normalizationprocess.org.

13. May C. Towards a general theory of implementation. Implement Sci. 2013;8:18-34.

14. May CR, Cummings A, Girling M, et al. Using Normalization Process Theory in feasibility studies and process evaluations of complex healthcare interventions: a systematic review. Implement Sci. 2018;13(80).

15. Francis JJ, Johnston M, Robertson C, et al. What is an adequate sample size? Operationalising data saturation for theory-based interview studies. Psychol Health 2010;25(10):1229-1245

16. Smith R. Audit, and research. BMJ. 1992;305(7489):905-906.

17. Creswell J. Qualitative Inquiry and Research Design: Choosing Among Five Approaches. second ed. Thousand Oaks: Sage Publications; 2007.

18. Gallacher K, May CR, Montori VM, Mair FS. Understanding patients' experiences of treatment burden in chronic heart failure using normalization process theory. Ann Fam Med. 2011;9(3):235-243.

19. Watson R, Parr JR, Joyce C, May C, Le Couteur AS. Models of transitional care for young people with complex health needs: a scoping review. Child Care Health Dev. 
2011;37:780-791.

20. May CR, Finch TL, Cornford J, et al. Integrating telecare for chronic disease management in the community: what needs to be done? BMC Health Serv Res. 2011;11(1):131-142.

21. Gask L, Rogers A, Campbell S, Sheaff R. Beyond the limits of clinical governance? The case of mental health in English primary care. BMC Health Serv Res. 2008;8:63-73.

22. Smith J, Firth J. Qualitative data analysis: the framework approach. Nurse Res. 2011;18(2):52-62.

23. Travaglia JF, Westbrook MT, Braithwaite J. Implementation of a patient safety management system as viewed by doctors, nurses and allied health professionals. Health. 2009;13:277-296.

24. Sahota S, Hughes P, Barrass C, et al. Delivering electronic prescribing and medicines administration in challenging areas such as paediatrics and maternity at King's College Hospital NHS Foundation Trust. Clin Med. 2015;15(3):5-12.
25. Choo J, Hutchinson A, Bucknall T. Nurses' role in medication safety. J Nurs Manag. 2010;18(7):853-861

26. Redwood S, Rajakumar A, Hodson J, Coleman JJ. Does the implementation of an electronic prescribing system create unintended medication errors? A study of the sociotechnical context through the analysis of reported medication incidents. BMC Med Inf Decis Making. 2001;11:29-40.

27. Cresswell KM, Bates DW, Williams R, et al. Evaluation of medium-term consequence of implementing commercial computerized physician order entry and clinical decision support prescribing systems in two "early adopter" hospitals. J Am Med Inf Assoc. 2014;21:194-202

28. Cresswell KM, Mozaffar H, Lee L, Williams R, Sheikh A. Safety risks associated with the lack of integration and interfacing of hospital health information technologies: qualitative study of hospital electronic prescribing systems in England. BMJ Qual Saf. 2017;26(7):530-541. 